\title{
LAMINAR NATURAL CONVECTION IN AN OPEN CYLINDRICAL CAVITY
}

\author{
Prashant Goyal ${ }^{1}$, Juby Abraham ${ }^{2}$, Anil Kumar Sharma ${ }^{3}$, K. Velusamy ${ }^{4}$ and P. Chellapandi ${ }^{5}$ \\ ${ }^{1}$ Department of Mechanical Engineering, Birla Institute of Technology Goa Campus, Goa, INDIA \\ 2,3,4,5 Indira Gandhi Centre for Atomic Research, Kalpakkam, INDIA \\ Email : aksharma@igcar.gov.in
}

\section{ABSTRACT}

Laminar natural convection of air in an open cylindrical cavity has been investigated,for a wide range of geometrical parameters. The conservation equations of mass, momentum and energy in axi-symmetric cylindrical coordinates have been solved, using the CFD code FLUENT. Ideal gas assumption is invoked to account for density variation of air. An extended computational domain has been considered along with the cavity for imposition of proper boundary conditions. The numerical results have been validated with published solutions for rectangular open cavities. Air flow patterns and temperature distributions in the cylindrical cavity have been analyzed with main focus on heat flux distribution and average Nusselt number. It is seen that heat flux distributions in cylindrical cavities are significantly different from that in rectangular cavities. The Nusselt number is high in cavities with low radius values indicating cylindrical cavities are better than rectangular cavities in heat dissipation. Further, the average heat flux and hence Nusselt number decrease as cavity width increases, demonstrating shallow cavities are inefficient in heat dissipation. The average Nusselt number is seen to exhibit two distinct variations with respect to Rayleigh number $(R a)$. In the conduction regime, it varies as $R a .45$ and in the convective regime, it varies as Ra 0.15 .

Keywords: laminar natural convection, cylindrical open cavity, Navier-Stokes equations, non-Boussinesq approximation.

\section{Nomenclature}

$C P \quad$ Specific heat of air

$g \quad$ Acceleration due to gravity

$h \quad$ Fin spacing

$k \quad$ Thermal conductivity of air

$\mathrm{Nu} \quad$ Nusselt number, Eq. (6)

$p \quad$ Pressure

$q \quad$ Normalized heat flux

$r \quad$ radial coordinate

Ra Rayleigh number, $\beta g(T c-T \propto) h 3 / v \alpha$

Tc Fin surface temperature

$T \propto \quad$ Ambient air temperature

u Radial velocity component

$v \quad$ Axial velocity component

w Fin length

$z \quad$ Axial coordinate

\section{Greek Letters}

$\alpha \quad$ Thermal diffusivity of air

$\beta \quad$ Coefficient of volumetric expansion

$\mu \quad$ Dynamic viscosity of air

$v \quad$ Kinematic viscosity of air

$\rho \quad$ Density of air

$\theta \quad$ Normalized air temperature, $\{(T-T \propto) /(T c-T \propto)\}$

$\psi \quad$ Stream function

$\psi \quad$ Normalized stream function, $\psi / \alpha$

\section{INTRODUCTION}

Open cavity natural convection is encountered in various engineering systems, such as open cavity solar thermal receivers, uncovered flat plate solar collectors having rows of vertical strips, electronic chips, geothermal reservoirs, energy conservation in buildings, fire research and energy-saving household refrigerators etc. Natural convection cooling is preferred over forced convection due to its passive nature in a variety of 
nuclear reactor applications. A detailed review of literature indicates that both experimental and computational investigations have been performed to understand heat transfer characteristics of open cavities. These studies may be classified into that pertaining to square / tall cavities and to shallow cavities.

\section{A. Square / Tall cavities}

Penot [1], numerically investigated natural convection in square cavities with the back wall facing the aperture isothermally heated, for various orientations of the cavities. He observed unsteady solutions for Grashof number exceeding $10_{5}$ when the aperture faces upwards. Later, Chan and Tien [2] considered a square cavity with horizontal opening for a wide range of Rayleigh number varying from $10_{3}$ to $10_{9}$ with unit Prandtl number. The back wall is isothermally hot while the two horizontal walls are adiabatic in their numerical study. They found that at high Rayleigh numbers, the heat transfer results approach that of a vertical flat plate. Hess and Henze [3] measured natural convective velocity distribution of air in a square cavity with horizontal opening, employing Laser-Doppler velocimetry at Rayleigh number between $3 \times 10_{10}$ and $2 \times 10_{11}$. Their experiments include unconstrained cavity (fully open) and constrained cavity with aperture ratio of 0.5 , with focus being boundary layer transition. Elsayed and Chakroun [4] experimentally investigated natural convection of air in square cavities with constrained and unconstrained apertures for various angles of tilt. They also varied the position of the aperture from bottom to top. High wall slit is found to transfer more heat to the surroundings than low wall slit.

They presented detailed correlations for Nusselt number in terms of opening ratio, tilt angle and slit position. Bilgen and Oztop [5] reported a comprehensive study of natural convection in partially open inclined square cavities. They identified the parameters for maximum and minimum heat transfer, in terms of aperture size and its position. Nouanegue et al. [6] investigated the effects of radiation on natural convection in square and tall open cavities. Bilgen and Muftuoglu [7] studied the effects of heating the back wall by constant heat flux and providing multiple slots in the aperture.
Sernas and Kyriakides [8] performed experiments in a horizontally open square cavity with all the three walls heated isothermally, at a Grashof number of $10_{7}$. The heat transfer rates along the back wall and top wall were measured using a Wollaston prism schlieren interferometer. Miyamoto et al. [9] numerically investigated natural convection in constrained and unconstrained square cavities at various tilt angles, with all the three walls isothermally heated. In this case, they found that the average Nusselt number is about half of that in the case of hot back wall with adiabatic top and bottom walls. Similarly, the dimensionless flow rate through the cavity is about twice that with hot back wall and adiabatic top and bottom walls.

\section{B. Shallow Cavities}

Quere et al. [10] numerically studied natural convection in shallow cavities with unconstrained aperture. In their study, the back wall is maintained hot while the other two walls of the cavity are adiabatic. Mohamad [11] studied natural convection in open cavities and slots with similar boundary conditions. This study covered various inclination angles $\left(10^{\circ}-90^{\circ}\right)$ and aspect ratios. It is found that the average Nusselt number is not very sensitive to inclination angle. In a recent study, Mohamad et al. [12] investigated shallow cavities of aspect ratio upto 10 , employing Lattice Boltzmann method for air. Chan and Tien [13-14] have studied natural convection of water in shallow cavity of aspect ratio 7 and provided data on Nusselt number.

Chakroun et al. [15] measured heat transfer coefficients in partially / fully open titled cavities at a flux Grashof number of $5.5 \times 108$. In their experiments, the back wall is supplied with a constant heat flux while other two walls are insulated, for aspect ratio in the range of 1.0 to 4.0. Polat and Bilgen [16] numerically studied natural convection in fully open shallow rectangular cavities for a wide range of Rayleigh number and cavity aspect ratio 1.0 to 8.0 . In this case also, the back wall is supplied with constant flux while other walls are insulated.

From the above literature survey, it is evident that only rectangular cavities have been studied and little attention has been paid to cylindrical open cavities. During the inward penetration of cold ambient fluid inside a cylindrical cavity, the flow area decreases. Further, the heat transfer through the boundary layer developing on the cylindrical stem is much different 
from that over a vertical plate. These differences can lead to significant changes in heat transfer features, compared to that in rectangular cavities, especially when the radius of the cavity is small. Hence, it is very essential to investigate the heat transfer features in open-ended cylindrical cavities, which is the focus of the present study.

\section{GOVERNING EQUATIONS AND SOLUTION METHOD}

Steady state non-Boussinesq form of conservation equations that govern the fluid flow and heat transfer processes in the cavity in axi-symmetric cylindrical coordinate system are [17]:

Continuity Equation:

$$
\frac{1}{r} \frac{\partial(\rho r u)}{\partial r}+\frac{\partial(\rho w)}{\partial z}=0
$$

Radial Momentum Equation:

$\rho\left(u \frac{\partial u}{\partial r}+w \frac{\partial u}{\partial z}\right)=\frac{\partial p}{\partial r}+\mu\left[\frac{1}{r} \frac{\partial}{\partial r}\left(\frac{r \partial u}{\partial r}\right)-\frac{u}{2}+\frac{\partial^{2} u}{\partial z^{2}}\right]$

Axial Momentum Equation:

$\rho\left(u \frac{\partial w}{\partial r}+w \frac{\partial w}{\partial x}\right)=\frac{-\partial p}{\partial z}+\mu\left[\frac{1}{r} \cdot \frac{\partial}{\partial r}\left(r \cdot \frac{\partial w}{\partial r}\right)+\frac{\partial^{2} w}{\partial z^{2}}\right]+g \ldots[3]$

Energy Equation:

$\rho C_{p}\left(u \cdot \frac{\partial T}{d e r}+w \frac{\partial T}{\partial z}\right)=k\left[\frac{1}{r} \frac{\partial}{\partial r}\left(r \frac{\partial T}{\partial r}\right)+\frac{\partial^{2} T}{\partial z^{2}}\right]$

In the above equations, $r$ is radial coordinate, $z$ is axial coordinate, $u$ is radial velocity component, $w$ is axial velocity component, $g$ is acceleration due to gravity, $T$ is fluid temperature, $\rho$ is density, $\mu$ is dynamic viscosity, $C P$ is specific heat of fluid and $k$ is fluid thermal conductivity.

\section{A. Boundary Conditions}

The computational domain along with boundary conditions is depicted in Fig. 1. The top, bottom and vertical walls of the cavity are maintained at constant temperature $T c$. The value of $T c$ is varied in the range of $333 \mathrm{~K}$ to $773 \mathrm{~K}$. The flow is laminar and the ambient temperature is $313 \mathrm{~K}$. The outer boundaries of the domain are extended 6 to four times the fin spacing (h) to make them sufficiently far from the main computational enclosure.

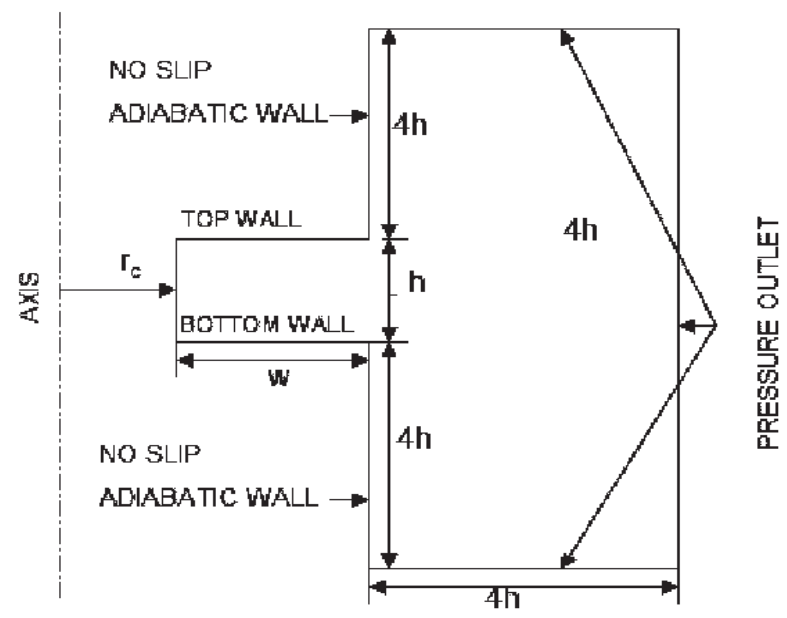

Fig. 1. Computational domain and boundary conditions

\section{B. Solution Method}

The conservation equations (1)-(4) are numerically solved by finite volume method [18] using the commercial CFD code FLUENT [19]. Pressure-velocity coupling in the above equations is resolved by the PISO algorithm [20]. The second order UPWIND scheme is used to combine the convective and diffusive fluxes in the momentum and energy equations.

\section{GRID INDEPENDENCE STUDY}

Detailed grid independence study has been carried out to identify the optimum grid size. A non-uniform grid pattern that gives adequate grid refinement close to the solid surfaces is used. A typical grid pattern is shown in Fig. 2. To test grid independence of the numerical scheme, solutions have been obtained on three non uniform mesh patterns with total number of grids 8776,11071 and 15016 . Table 1 shows the average Nusselt number for the three different mesh configurations for a case of $R a=105, r c / h=1$ and $w / h=2$. The difference in average Nusselt numbers between the mesh with 11071 nodes and 8776 nodes is $1 \%$, while that between 11071 nodes and 15016 nodes is only $0.04 \%$. Hence, a grid size of 11071 nodes has been used in all the subsequent computations. 


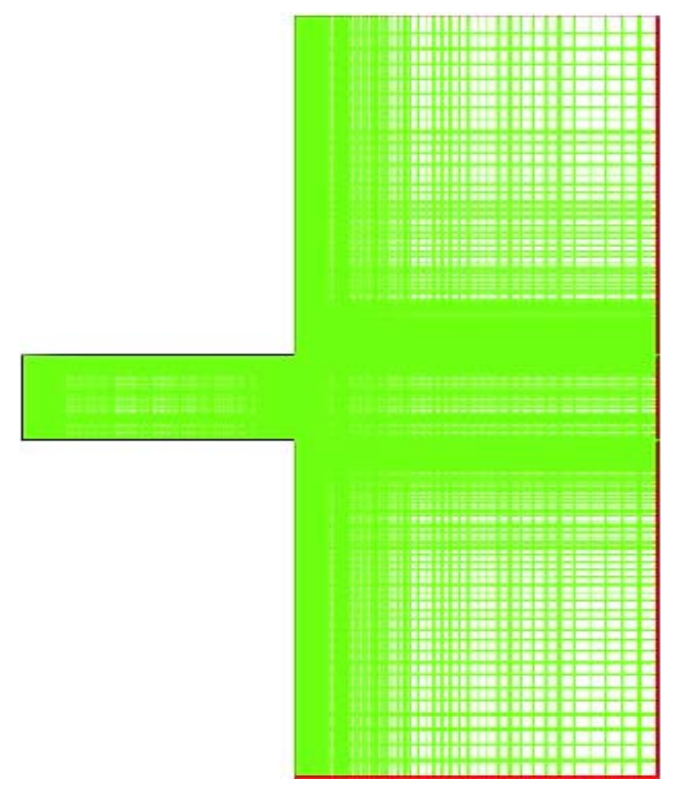

Fig. 2. Computational grid pattern

Table 1. Results of grid independence study at $R a=10_{5}$ and $r_{c} / h=1$ and $w / h=2$

\begin{tabular}{|c|c|c|c|}
\hline SI No. & $\begin{array}{c}\text { Number of } \\
\text { Nodes }\end{array}$ & $\begin{array}{c}\text { Average } \\
\mathrm{Nu}\end{array}$ & $\%$ Change \\
\hline 1 & 8776 & 4.39 & - \\
\hline 2 & 11071 & 4.43 & 1 \\
\hline 3 & 15016 & 4.45 & 0.04 \\
\hline
\end{tabular}

\section{VALIDATION}

The present computational model has been validated against published results of Chan and Tien [13], for side open square cavities at various Rayleigh numbers with a constant Prandtl number of unity. The present results of Nusselt number are compared in Table 2 along with the published data. It can be seen that the 7 comparison is highly satisfactory, with a maximum deviation less than $2 \%$, validating the present computational procedure.

Table 2. Comparison of predicted Nusselt number with published data

\begin{tabular}{|c|c|c|c|}
\hline$R a$ & $N u$ (Chan and Tien [13]) & $N u$ (present) & $\%$ Error \\
\hline $10_{3}$ & 1.07 & 1.09 & 1.87 \\
\hline $10_{4}$ & 3.41 & 3.46 & 1.47 \\
\hline $10_{5}$ & 7.69 & 7.76 & 0.91 \\
\hline $10_{6}$ & 15.00 & 15.20 & 1.33 \\
\hline
\end{tabular}

\section{RESULTS AND DISCUSSION}

As mentioned earlier, the focus of this paper is fluid flow and heat transfer from axi-symmetric open cavities formed by cylindrical fins. The fin spacing $(h)$, fin width ( $w$ ) and radius of the stem to which the fins are attached $(r c)$ have been varied from $15 \mathrm{~mm}$ to 45 $\mathrm{mm}$. The Rayleigh number (Ra) based on fin spacing is varied from 103 to 106 . The number of parametric studies carried out is 108 .

\section{A. Comparison of Cylindrical and Rectangular Open Cavities}

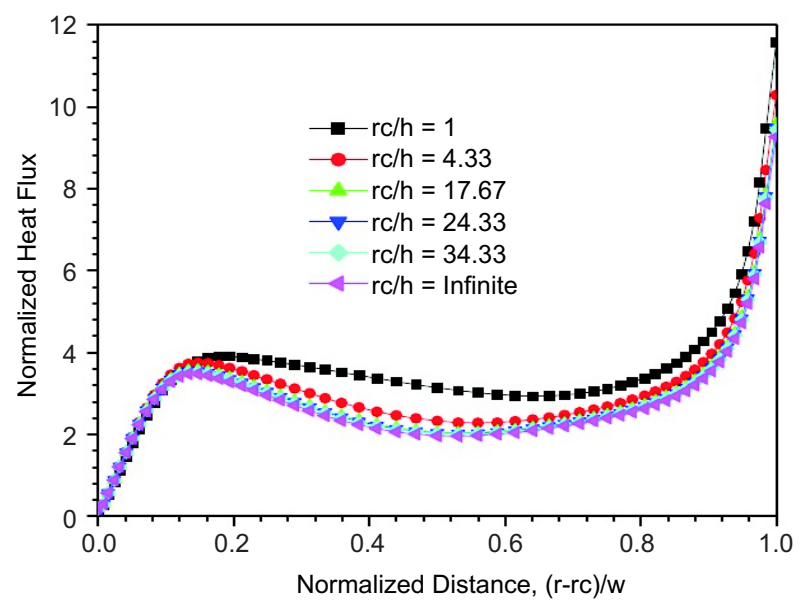

Fig. 3. Heat flux distribution on top wall of the cavity as function of $r_{c} / h$ for $R a=10_{5}$ at $w / h=2$

Figure 3 depicts the distribution of non-dimensional heat flux on top wall of the cavity as function of normalized distance $(r-r c) / h$ for a specific Rayleigh number of 105 at $w / h=2$. The heat flux is normalized using the following relationship,

$$
q=-k\left|\frac{\partial T}{\partial z}\right|_{z=h} /\{k(T c-T \propto / h\}
$$

Heat flux $(q)$ increases linearly upto $10 \%$ of the cavity width where the wall jet developing adjacent to the stem (i.e., vertical wall) impinges on top wall. Then it decreases upto $60-70 \%$ of the cavity width. Beyond this point, the flux again increases for remaining portion on the wall. This increase is due to the reduction in air temperature as a consequence of mixing between hot cavity fluid emanating outwards and cold ambient fluid. As the non-dimensional radius, $r c / h$, increases the heat flux reduces. This reduction is only upto $r c / h \sim 20$ and beyond this radius, the flux distribution approaches that of a rectangular cavity, demonstrating 
that the curvature effects are significant upto $r c / h=20$. Thus, it is clear that the correlations established for rectangular cavities are not applicable for cylindrical cavities and independent studies are essential for cylindrical cavities, which is the objective of this study.

Average Nusselt number for the entire cavity $(\mathrm{Nu})$ defined as,

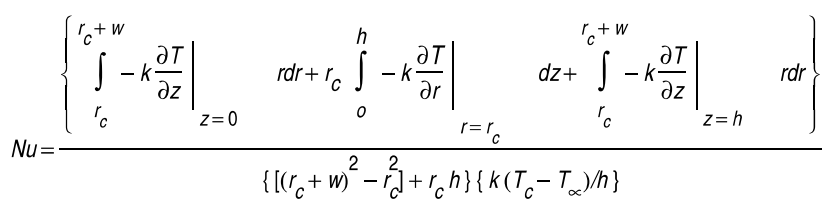

as a function of $r c / h$ for Rayleigh number of 105 and $w / h=2$ is depicted in Fig. 4. On comparing the results for $r c / h=1$ and $r c / h=20$, it is clear that as radius decreases, the Nusselt number increases by as much as $15 \%$. These results justify that the $\mathrm{Nu}$ number for cylindrical cavities are different from that of rectangular cavities. In the subsequent subsections results of cylindrical cavities are highlighted.

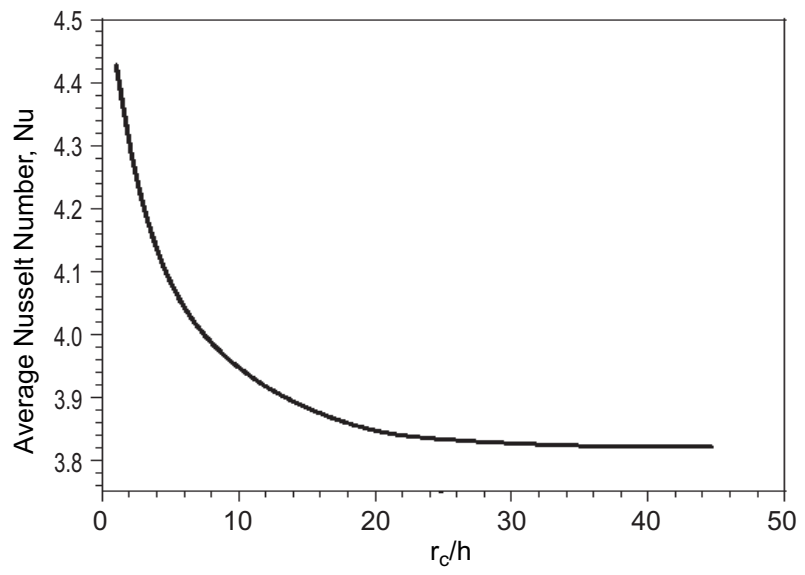

Fig. 4. Variation of $N u$ as function of $r_{d} / h$ for

$$
R a=105 \text { at } w / h=2
$$

\section{B. Effect of Rayleigh Number}

The distribution of streamlines and isotherms for two different $R a$ numbers at $r c / h=1$ and $w / h=3$ are depicted in Figs. 5 (a) - (d). In these figures, the stream function $(\psi)$ and temperature are normalized as,

$$
\psi=\psi / a \& \theta=(T-T \propto) /(T c-T \propto)
$$

At low $R a$ values (Figs. $5(\mathrm{a})$ ), convection is very weak. The ambient fluid does not penetrate much into the cavity and the mild convection is restricted to the mouth of the cavity. From isotherms depicted in Fig. 5 (b), it is evident that the cavity fluid is hot and is nearly at the temperature of the fin. This suggests that the cavity air acts as an insulator rather than heat transport medium at low Rayleigh numbers.

However, as the Ra increases, natural convection becomes increasingly vigorous. The natural convective flow rate inside the cavity is represented by the difference in the magnitudes of streamlines ( $\Delta \psi=\psi \max -\psi \min$ ). The value of $\Delta \psi$ for $R a=10_{3}$ is 0.3 and the same for $R a=10_{6}$ is 7 . The ratio between these two values is 23 . This 9 suggests that the magnitude of natural convective flow rate scales as $(R a)_{0.20}$ in cylindrical cavities. In rectangular cavities, the flow rate is expected to scale as $(R a)_{0.5}$. In Fig. 5 (c) it is seen that the streamlines are more or less uniformly spaced while entering the cavity. This suggests that the flow enters the cavity with a fairly uniform velocity. While leaving, the streamlines are more densely packed than at the inlet. Hence, the air velocity leaving the cavity is higher than the inlet value. In Fig. 5 (d), it is evident that the cavity is cold and hence there is significant increase in heat exchange between the hot wall and the cold fluid, at high $R a$ numbers.

\section{Effect of Cavity Width}

To understand the influence of cavity width on heat transfer characteristics, streamlines and isotherms at $R a=10_{5}$ and $r c / h=1$ for two different values of $w / h$ are depicted in Figs. 6 (a) - 6 (d). On comparing $\Delta \psi$ values for $w / h=1$ and 3 , it is clear that the circulation rate of natural convection increases as cavity width increases. For example, it is 1.3 for $w / h=1$ and is 4.5 for $w / h=3$. The circulation rate increases almost linearly with $w / h$. On comparing the isotherms, it is clear that the temperature gradient adjacent to the vertical wall (stem) is less in the case of $w / h=3$ than that in the case of $w / h=1$. This suggests that the participation of vertical wall in heat dissipation is less effective in shallow cavities. In Figs. 6 (b) and 6 (d), it is clear that the isotherms are sparsely spaced around the top wall while they are closely packed near the bottom wall. This is due to the fact, when the fluid enters the cavity, it is cold and hence it absorbs maximum heat from the bottom wall. However, when the preheated air approaches the top wall it absorbs 


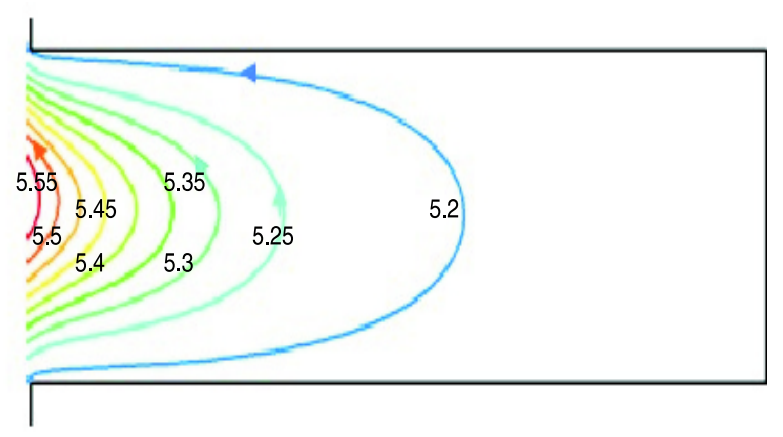

Fig. 5 (a) Streamlines for $R a=10^{3}, r_{c} / h=1$ and

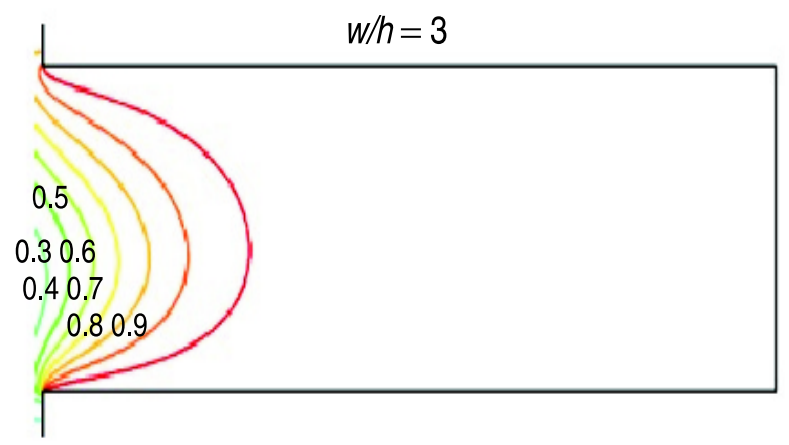

Fig. 5 (b) Isotherms for $R a=10^{3}, r c / h=1$ and

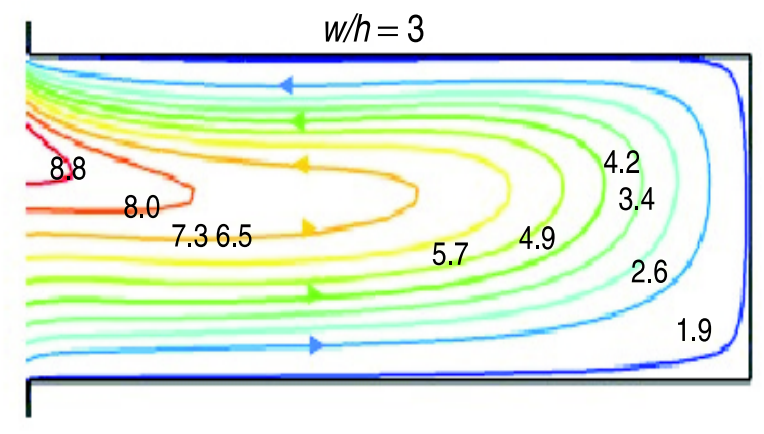

Fig. 5 (c) Streamlines for $R a=10^{6}, r_{c} / h=1$ and

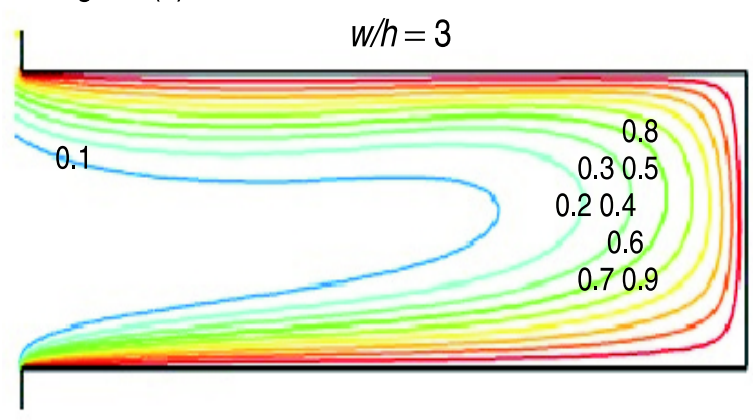

Fig. 5 (d) Isotherms for $R a=10^{6}, r_{c} / h=1$ and

$$
w / h=3
$$

less heat. Hence, heat dissipation is expected to be more from the bottom wall than from top wall.

\section{Effect of Cavity Radius}

The streamlines and isotherms for two different values of stem radius, viz. $r c / h=1$ and 3 , at identical values of $R a$ and $w / h$ are depicted in Figs. 7 (a) - 7 (d). It is clear from these figures that as the radius increases, the natural convective flow rate inside the cavity increases. For example, at $r c / h=1$, the value of $\Delta \psi$ is 2.39 , which increases to 4.20 at $r c / h=3$. However, the isotherm patterns are nearly identical.

\section{E. Non-dimensional Heat Flux}

Figure 8 depicts normalized heat flux distribution along the cavity walls for two different $R a$ numbers. Along bottom wall, the flux is maximum when cold fluid just enters the cavity, due to small thickness of thermal boundary layer. The flux monotonically decreases towards the stem (i.e., upto vertical wall), due to growth of boundary layer and increase in fluid temperature. Along the top wall, flux is high at a location where the vertical wall jet impinges. Beyond this point, the flux decreases due to boundary layer growth and stable stratification. However, at the cavity mouth, it again increases due to mixing between the outgoing hot fluid and the cold ambient fluid. On the vertical wall, the flux is maximum at mid-height and it decreases towards the corners due to near stagnant flow conditions.

Effect of cavity width on flux distribution is depicted in Fig. 9. Comparing the results of $w / h=1$ and 3 , it is clear that flux is low in the case of shallow cavities $(w / h=3)$. In other words, shallow cavities are less efficient in heat dissipation, due to limited availability of fresh fluid for parts of wall surfaces away from the mouth. Among the three walls, the vertical wall is the most affected one. Figure 10 depicts the effect of cavity radius on flux distribution. As the radius decreases, flux decreases on bottom wall and vertical wall. Contrary to this, flux increases on the top wall as radius decreases.

\section{F. Average Nusselt Number}

Variation of average $\mathrm{Nu}$ number (defined in Eq. 6) as a function of Ra number and cavity geometrical parameters, viz., radius, width and height, is depicted in Fig. 11. In the conduction regime, the Nu number is even lower than unity, especially in shallow cavities. Convection effects assume significance beyond $\mathrm{Ra}$ 


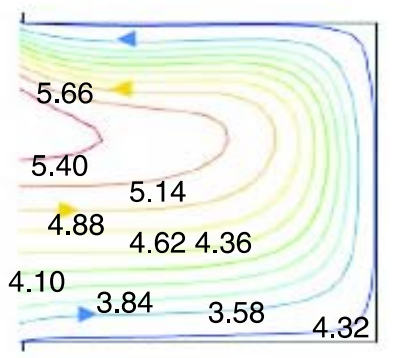

Fig. 6 (a) Streamlines for $R a=10^{5}, r_{c} / h=2$ and $w / h=1$

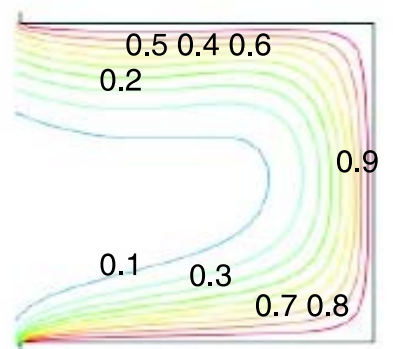

Fig. 6 (b) Isotherms for $R a=10^{5}, r_{c} / h=2$ and $w / h=1$

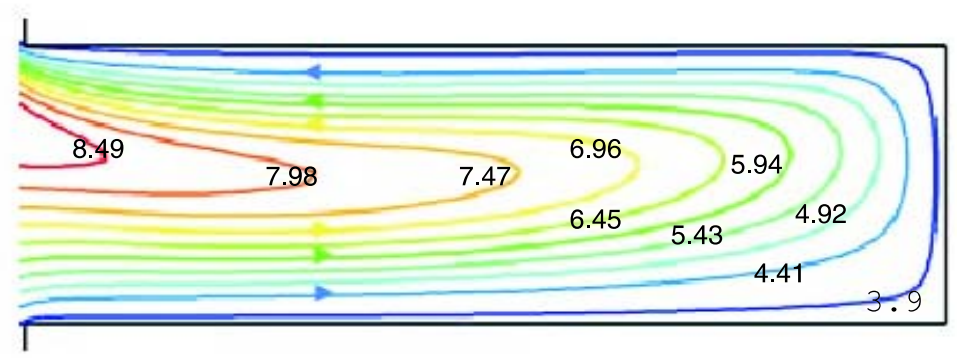

Fig. 6 (c) Streamlines for $R a=10^{5}, r d h=2$ and $w / h=3$

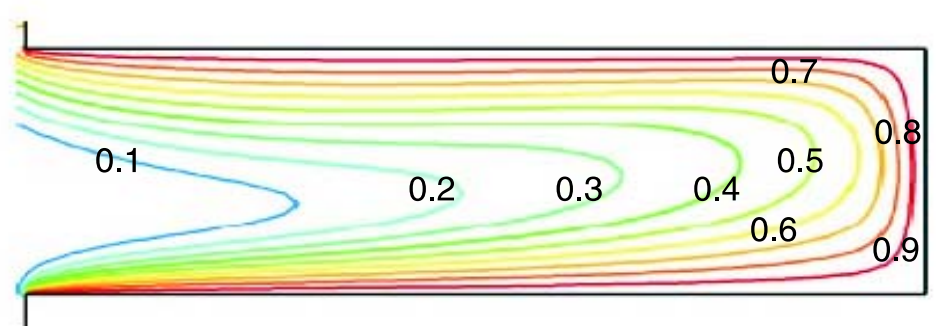

Fig. 6 (d) Isotherms for $R a=10^{5}, r_{c} / h=2$ and $w / h=3$

$=2 \times 10_{4}$. As discussed in the earlier sections, $\mathrm{Nu}$ number is low when either cavity radius or cavity width is high. In all the cases analyzed, the $N u$ number is seen to exhibit two distinctly different variations with respect to Rayleigh number. In the conduction dominated regime, viz., $\mathrm{Ra} \leq 10_{4}, \mathrm{Nu}$ exhibits a strong variation with respect to $R a$, as $R a 0.45$. In the convection dominated regime, viz., $R a \geq 10_{5}$, it increases as $R a 0.15$.

\section{CONCLUSION}

Laminar natural convection of air in cylindrical open cavities has been investigated, for a wide range of geometrical parameters. The 2-D conservation equations of mass, momentum and energy in 


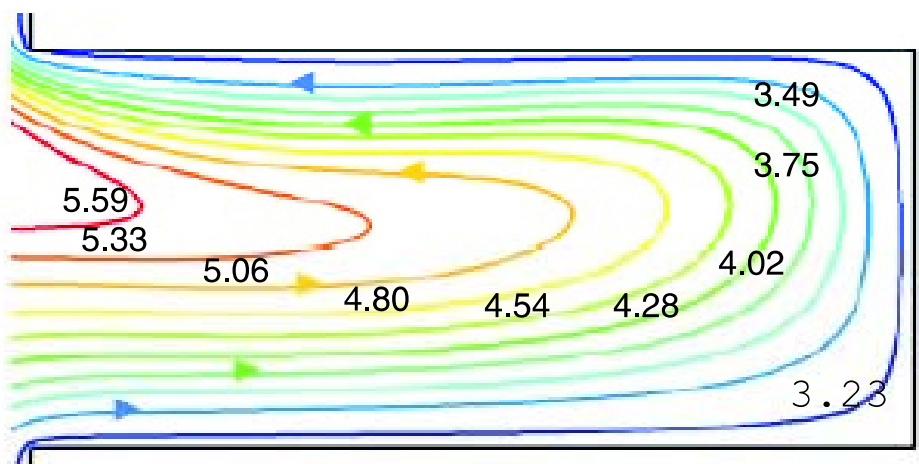

Fig. 7 (a) Streamlines for $R a=10^{4}, r d h=1$ and $w / h=2$

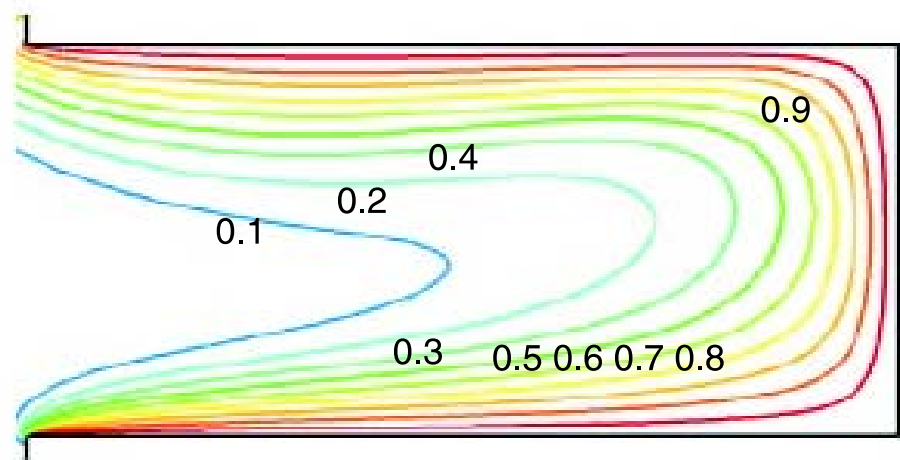

Fig. 7 (b) Isotherms for $R a=10^{4}, r_{c} / h=1$ and $w / h=2$

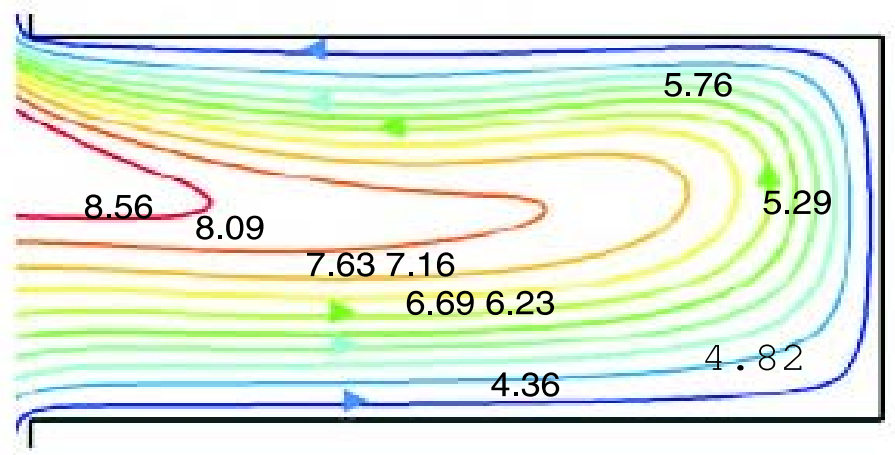

Fig. 7 (c) Streamlines for $R a=10^{4}, r_{c} / h=3$ and $w / h=2$

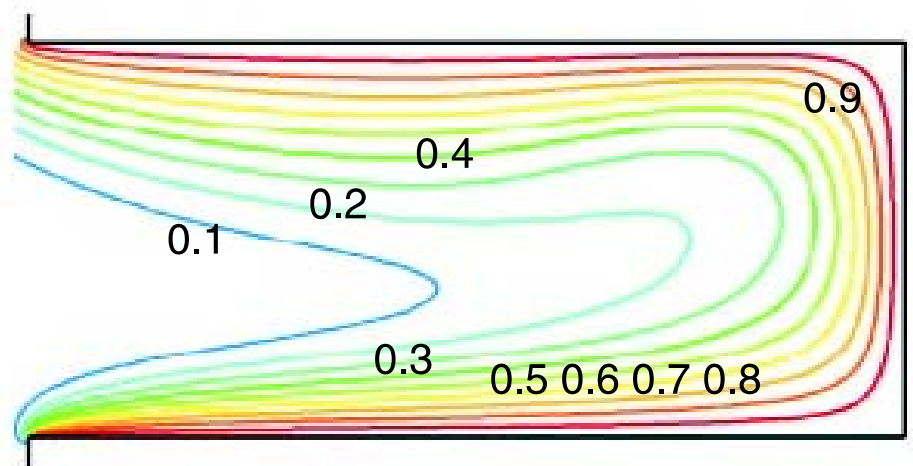

Fig. 7 (d) Isotherms for $R a=104, r d h=3$ and $w / h=2$ 


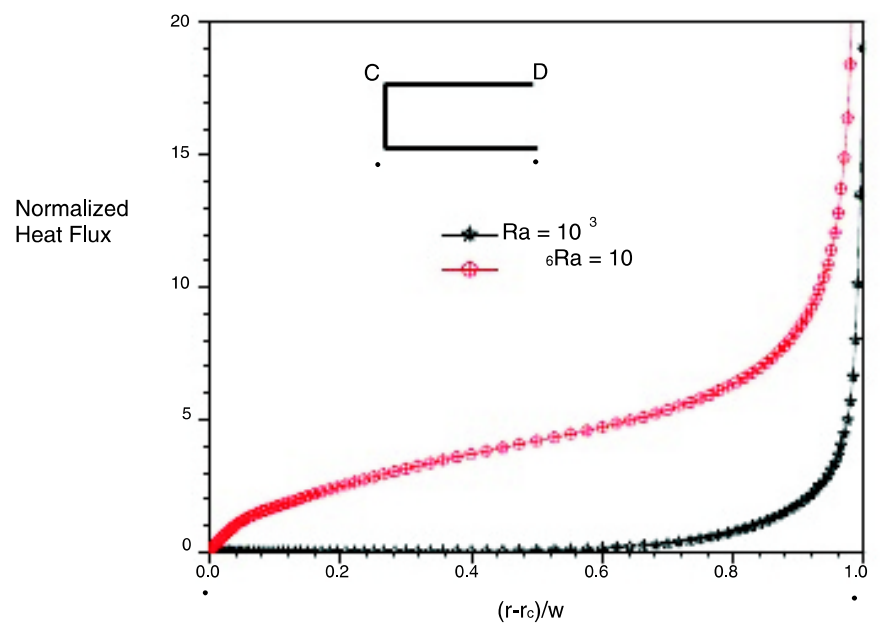

Fig. 8 (a) Heat flux on bottom wall $\left(R a=10^{3}\right.$ and $10^{6}, r_{c} / h=1$ and $\left.w / h=3\right)$

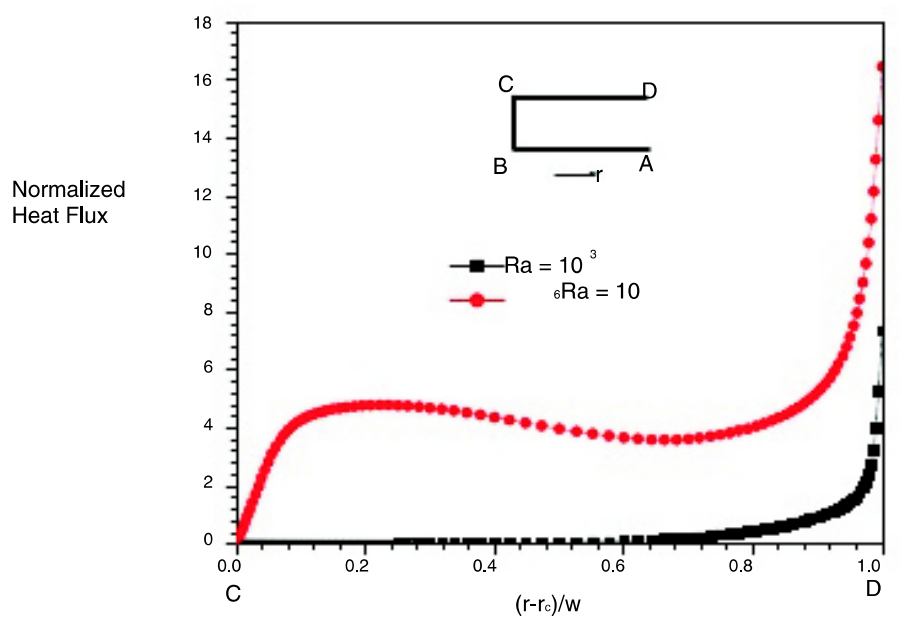

Fig. 8 (b) Heat flux on top wall $\left(R a=10^{3}\right.$ and $10^{6,} r c / h=1$ and $\left.w / h=3\right)$

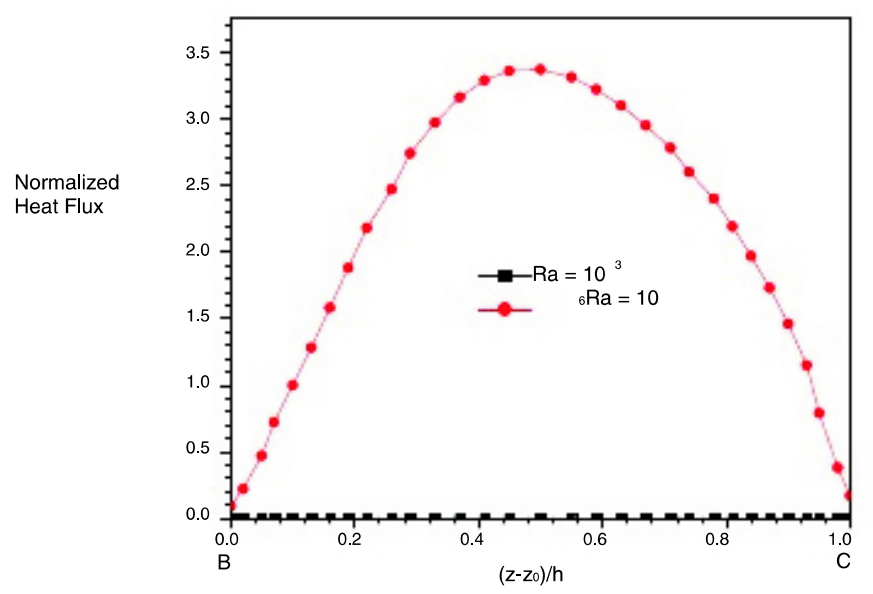

Fig. 8 (c) Heat flux on vertical wall $\left(R a=10^{3}\right.$ and $10^{6}, r c / h=1$ and $\left.w / h=3\right)$ 


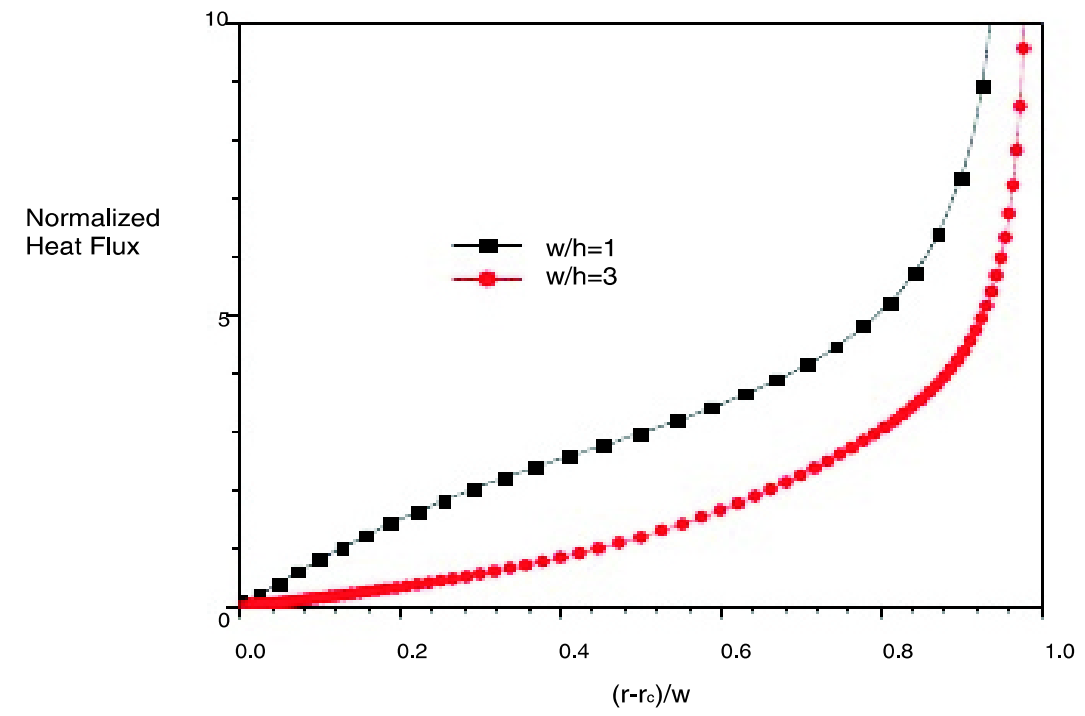

Fig. 9 (a) Heat flux on bottom wall $\left(R a=10^{4}, r_{c} / h=2, w / h=1 \& 3\right)$

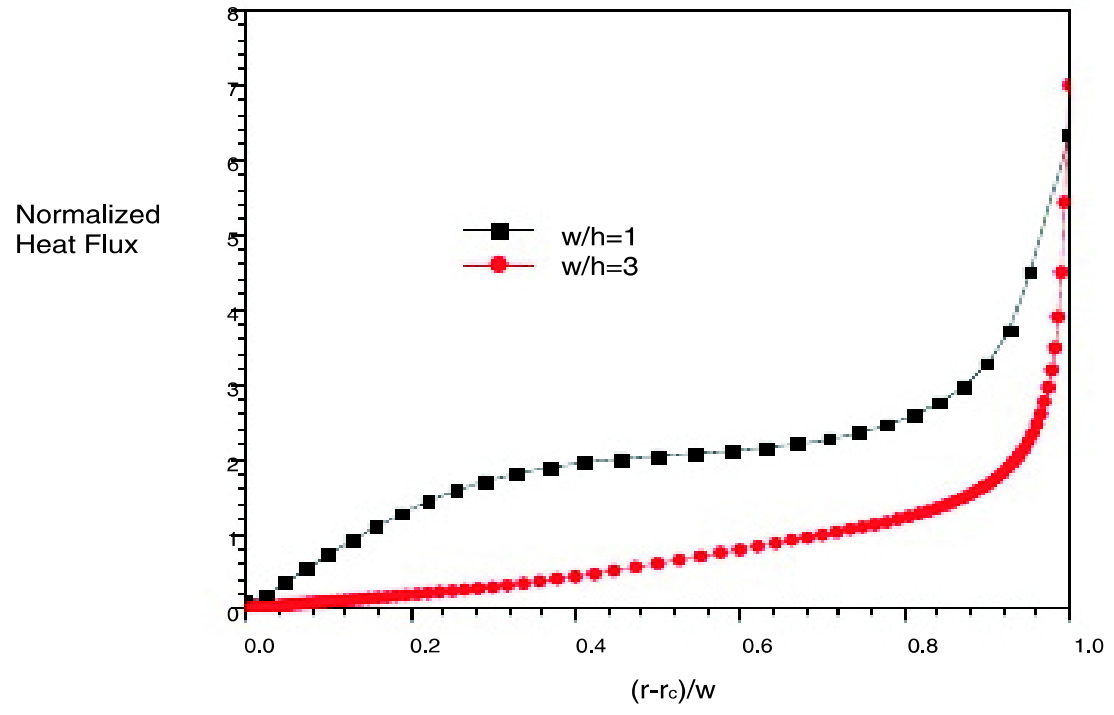

Fig. 9 (b) Heat flux on top wall $\left(R a=10^{4}, r d h=2, w / h=1 \& 3\right)$

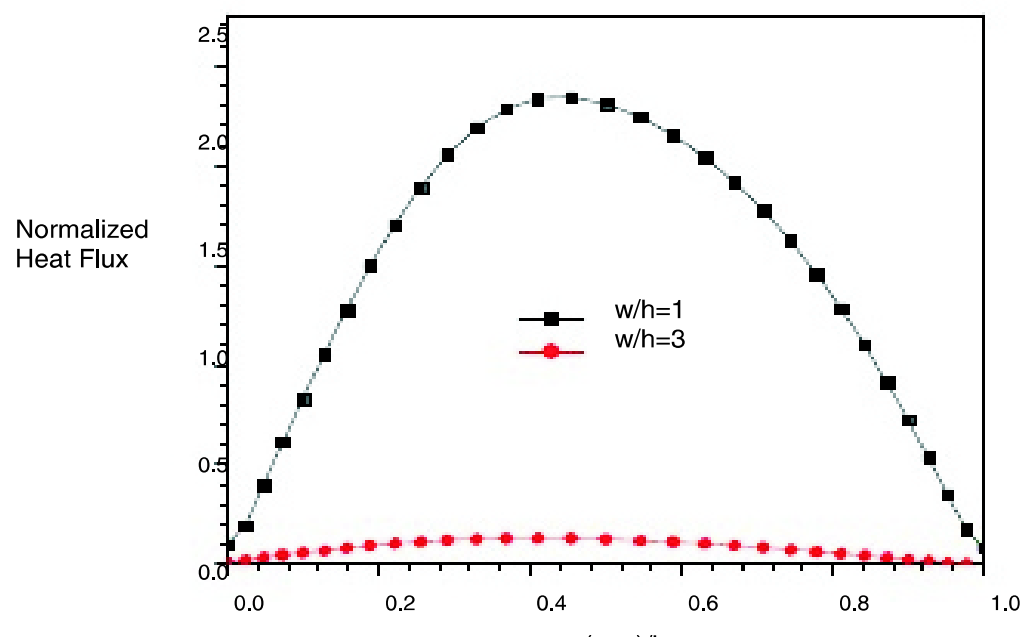

Fig. 9 (c) Heat flux on vertical wall $\left(R a=10^{\left(z-z_{0}\right) / h}, r_{c} / h=2, w / h=1 \& 3\right)$ 


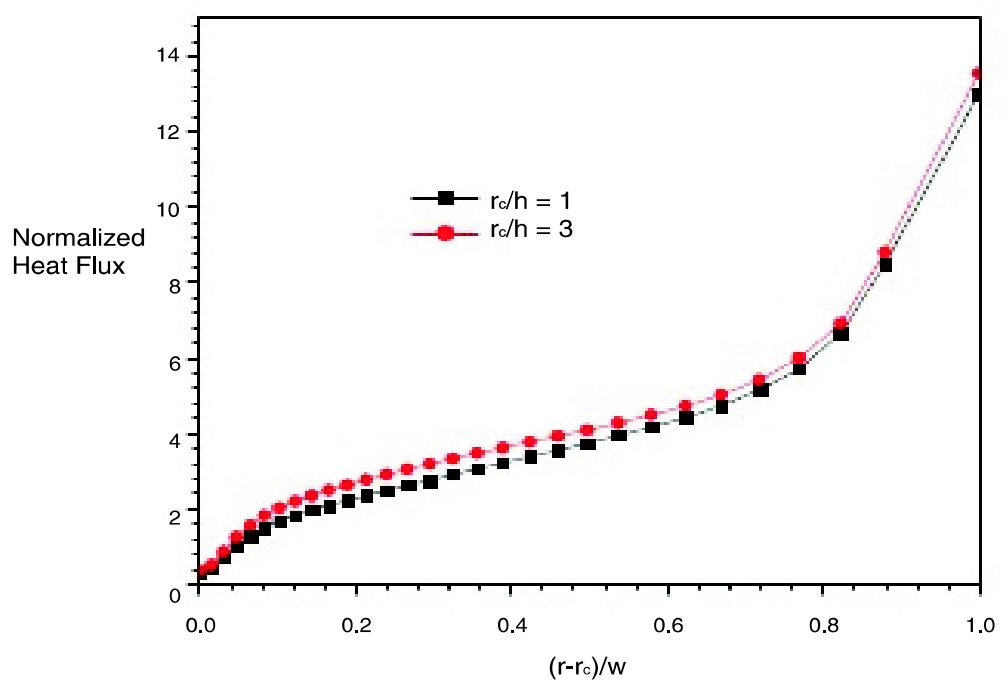

Fig. 10 (a) Heat flux on bottom wall $\left(R_{a}=10^{5}, r d h=1 \& 3\right.$ and $\left.w / h=2\right)$

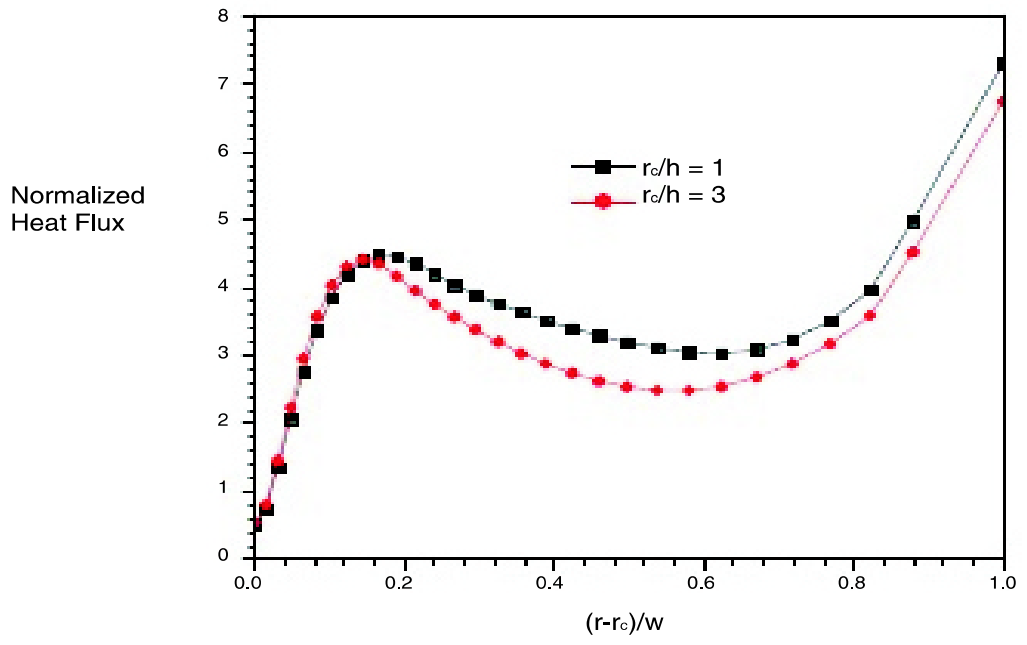

Fig. 10 (b) Heat flux on top wall $\left(R a=10^{5}, r_{c} / h=1 \& 3\right.$ and $\left.w / h=2\right)$

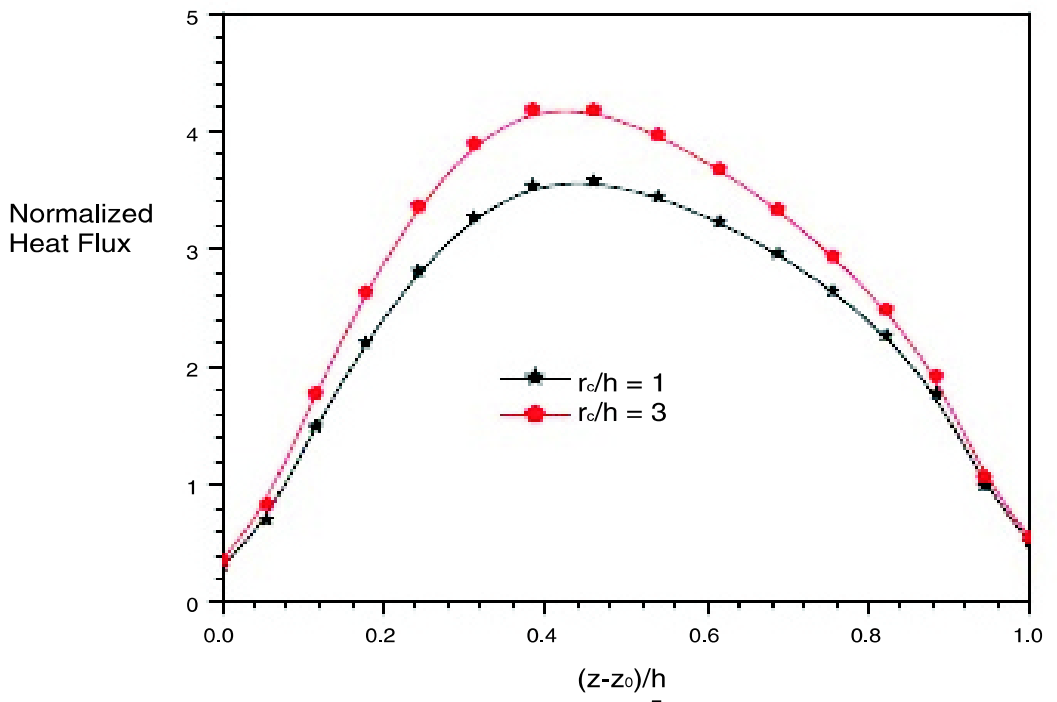

Fig. 10 (c) Heat flux on vertical wall $\left(R a=10^{5}, r_{c} / h=1 \& 3\right.$ and $\left.w / h=2\right)$ 


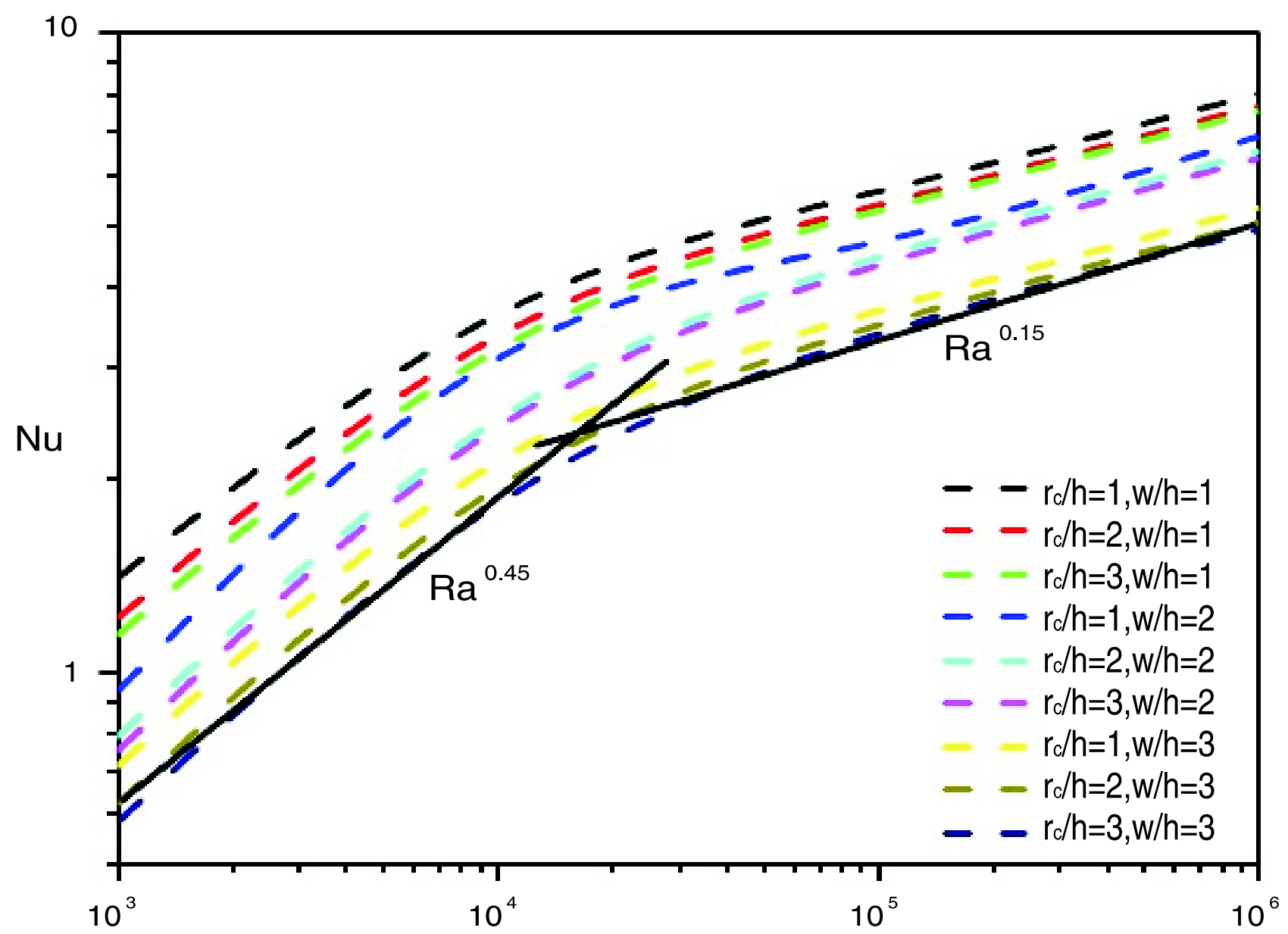

$\mathrm{Ra}$

Fig. 11 Variation of $\mathrm{Nu}$ number with Rayleigh number

axi-symmetric cylindrical coordinates have been solved, numerically using the CFD code FLUENT. An extended domain has been considered along with the cavity for imposition of proper boundary conditions. The numerical solutions have been validated against published solutions for rectangular open cavities. Air flow patterns and temperature distributions in the cavity have been analyzed with the main focus on heat flux distribution and average Nusselt number. It is seen that heat flux distributions in cylindrical cavities are significantly different from that in rectangular cavities. Heat flux is more at the bottom wall than at other two walls. The Nu number is high if the cavity radius is high, indicating cylindrical cavities are better than rectangular cavities in heat dissipation. Further, the heat flux and hence Nu number decreases as cavity width increases, demonstrating shallow 12 cavities are not efficient in heat dissipation. The Nu number exhibits two distinct variations with respect to $R a$ number. In the conduction regime, it varies as $R a 0.45$ and in the convective regime, it varies as $R a 0.15$.

\section{REFERENCES}

[1] Penot.F., 1982, "Numerical calculation of two-dimensional natural convection in isothermal open cavities", Numerical Heat Transfer Part-A 5 421-437.

[2] Chan Y.L and Tien C.L., 1986 "A numerical study of two-dimensional natural convection in square open cavities", Numerical Heat Transfer Part-B 8 65-80.

[3] Hess C.F and Henze R.H., 1984 "Experimental investigation of natural convection losses from open cavities", Journal of Heat Transfer 106, 333-338.

[4] Elsayed M.M and Chakroun W., 1999" Effect of aperture geometry on heat transfer in tilted partially open cavities", Journal of Heat Transfer 121, 819-827. 
[5] Bilgen E and Oztop H., 2005 "Natural convection heat transfer in partially open inclined square cavities", International Journal of Heat and Mass Transfer 48, 1470-1479.

[6] Nouanegue H., Muftuoglu A and Bilgen E., 2008 "Conjugate heat transfer by natural convection, conduction and radiation in open cavities", International Journal of Heat and Mass Transfer 51, 6054-6062.

[7] Bilgen E and Muftuoglu A., 2008 "Natural convection in an open square cavity with slots", International Communications in Heat and Mass Transfer 35, 896-900.

[8] Sernas V and Kyriakides I., 1982 "Natural convection in an open cavity", Proceedings of seventh Heat Transfer Conference, Munich, pp. 275-286.

[9] Miyamoto M., Kuehn T.H., Goldstein R.J and Katoh Y., 1989 "Two dimensional laminar natural convection heat transfer from a fully or partially open square cavity", Numerical Heat Transfer Part- A 15, 411-430.

[10] Quere P.L., Humphrey J.A.C and Sherman F.S., 1981 "Numerical calculation of thermally driven two-dimensional unsteady laminar flow in cavities" of rectangular cross section, Numerical Heat Transfer Part-A 4, 249- 283.

[11] Mohamad A.A., 1995 "Natural convection in open cavities" and slots, Numerical Heat Transfer Part-A 27, 705-716.
[12] Mohamad A.A., El-Ganaoui M and Bennacer R., 2009 "Lattice Boltzmann simulation of natural convection in an open ended cavity", International Journal of Thermal Sciences 48, 1870-1875.

[13] Chan Y.L and Tien C.L., 1985 "A numerical study of two-dimensional laminar natural convection in shallow open cavities", International Journal of Heat and Mass Transfer 8, 603-612.

[14] Chan Y.L and Tien C.L., 1986 "Laminar natural convection in shallow open cavities", Journal of Heat Transfer 108, 305-309.

[15] Chakroun W., Elsayed M.M and Al-Fahed S.F., 1997 "Experimental measurements of heat transfer coefficient in a partially / fully opened tilted cavity", Journal of Solar Energy Engineering 119, 298-303.

[16] Polat $O$ and Bilgen E., 2002 "Laminar natural convection in inclined open shallow cavities", International Journal of Thermal Sciences 41, 360368.

[17] Bird R.B., Stewart W.E and Lightfoot E.N., 2005 "Transport Phenomena", John-Wiley \& Sons, New York, 2nd Edition.

[18] Patankar S.V., 1980 "Numerical Heat Transfer and Fluid Flow", Hemisphere.

[19] Fluent Inc. 1998, User's guide, Vol. 1-5.

[20] Versteeg H.K and Malalasekara W., 1995 "An Introduction to Computational Fluid Dynamics" - The Finite Volume Method, John Wiley \& Sons, New York. 